\title{
Nonlinear regression applied for power quality disturbances characterization in grids with wind generators
}

\author{
P. Janik, T. Lobos, J. Rezmer, Z. Wacławek
}

Department of Electrical Engineering

Wroclaw University of Technology

Wybrzeze Wyspianskiego 27, 50-370 Wroclaw, Poland

Phone:+0048 3202901, przemyslaw.janik@pwr.wroc.pl

\begin{abstract}
The impact of wind generation on the electrical system should be assessed to figure out potential hazards to system operation and deterioration of power quality indicia. In this paper signal processing algorithms has been applied to analyze switching transients within wind generation units. Nonlinear regression method and Prony model were applied to determine transients' parameters for various operation modes of the wind generator. Both methods delivered quite satisfactory results, but the regression method was prone to local minima.
\end{abstract}

\section{Key words}

Please write the main key words of your text (3 to 6) separated by commas.

\section{Introduction}

The widespread implementation of wind energy conversion systems is a reality. Wind is seen as clean and renewable energy source, so the development of wind generation technologies is welcomed and supported by ecologists and governments. In the next years we will have even more generator units connected to the grid [1].

Besides distinctive merits, the connection of wind generators leads to many disturbances, such as: voltage fluctuation, flickers, harmonics, instability, blind power regulation problems, and transients [2]. Those disturbances affect power quality. Power quality issues connected with wind generation are not only important because of technical aspects, they are also crucial on the free energy market.

There are at least three main wind generators structures, which can be pointed out [3]. The simplest one, but certainly not advanced technologically, is the squirrelcage induction generator connected directly to the grid.

Many of the wind energy converters installed today still have a squirrel-cage induction machine connected directly to the grid $[4,5]$,. This type of the generator cannot perform voltage control and it absorbs reactive power from the grid. Compensating capacitors are often directly connected. That type of installation is cost saving, and therefore widely used, but from the system analysis point of view it can be considered as a potential source of disturbances [3].

During the switching of capacitors transients occur, which are devastating for sensitive equipment, protection relays and insulation. The impact of transients on power quality indices cannot be neglected [2]. Transient overvoltages can theoretically reach peak values up to $2.0 \mathrm{pu}$. High current transients can reach values up to ten times the nominal capacitor current with duration of several milliseconds [6].

The purpose of this paper is the assessment of transients in electrical system with asynchronous wind generator. The analysis was carried out for real measured signals and for different operation conditions of the wind converter simulated in Matlab in SimPowerSystem Toolbox [9].

Presented below, the nonlinear regression method and the Prony method were considered as appropriate tools for parameters estimation of transients. Further, the simulation model and analyses of simulated and measured signals are presented.

\section{Nonlinear regression}

Nonlinear regression is a general technique to fit a curve through data. It fits data to any equation that defines $Y$ as a function of $X$ and one or more parameters. It finds the values of those parameters that generate the curve that comes closest to the data (minimizes the sum of the squares of the vertical distances between data points and curve). That technique requires a model of the analyzed signal. In the case of capacitor bank switching, the signal model of transient component can be defined as

$$
x(t)=A_{1} \sin \left(\omega_{1} t+\varphi_{1}\right)+A_{2} e^{-\alpha t} \sin \left(\omega_{2} t+\varphi_{2}\right)
$$

where $A$ - amplitudes, $\alpha$ - damping factor and $\omega$ - angular velocities are unknown and should be estimated. 
Practically, the signal is observed (or measured) during a finite duration of time and $N$ samples of this signal are available. The measured discrete time signal $y\left(n T_{p}\right)$ can be specified as

$$
\begin{aligned}
& y\left(n T_{p}\right)=A_{1} \sin \left(\omega_{1} n T_{p}+\varphi_{1}\right)+ \\
& +A_{2} e^{\left(-\alpha n T_{p}\right)} \sin \left(\omega_{2} n T_{p}+\varphi_{2}\right)+e\left(n T_{p}\right)= \\
& x\left(n T_{p}\right)+e\left(n T_{p}\right)
\end{aligned}
$$

for $n=1,2,3 \ldots, N$.

$T_{p^{-}}$is the sampling period, $N$ - the number of samples and $e$ the estimation error correlated with each sample, which includes random noise and other distortions.

The problem of nonlinear regression can be formulated as an optimization problem, where the goal is to minimize the difference between the physical observation and the prediction from the mathematical model. More precisely, the goal is to determine the best values of the unknown parameters $A, \alpha, \omega, \varphi$ in order to minimize the squared errors between the measured values of the signal and the computed ones.

Thus, the optimization problem can be formulated as follows:

Find a vector $\mathbf{w}=\left[A_{1}, A_{2}, \alpha, \omega_{1}, \omega_{2}, \varphi_{1}, \varphi_{2}\right]^{T}$ which minimizes the objective function

$$
E(\mathbf{w})=\sum_{n=1}^{N} e^{2}\left(n T_{p}\right)=\sum_{n=1}^{N}\left[y\left(n T_{p}\right)-x\left(n T_{p}\right)\right]^{2}
$$

That is a well known standard least squares problem. To solve that problem, the Quasi-Newton method was applied [12]. At each iteration, the problem is to find a new iterate $w_{k+1}$ of the form:

$$
\mathbf{w}_{k+1}=\mathbf{w}_{k}+\tau \mathbf{d}
$$

where $\tau$ is a scalar step length parameter and $d$ is the search direction. Using the quasi-Newton method, a line search is performed in the direction

$$
\mathbf{d}=-\mathbf{H}_{k}^{-1} \cdot \nabla E\left(\mathbf{w}_{k}\right)
$$

where $\mathrm{H}^{-1}$ is an approximation of the inverse Hessian matrix.

The DFP formula was used for approximation of the inverse of Hessian matrix

$$
\mathbf{H}_{k+1}^{-1}=\mathbf{H}_{k}^{-1}+\frac{\mathbf{s}^{k}\left(\mathbf{s}^{k}\right)^{T}}{\left(\mathbf{q}^{k}\right)^{T} \mathbf{s}^{k}}-\frac{\left(\mathbf{H}_{k}^{-1} \mathbf{q}^{k}\right)\left(\mathbf{H}_{k}^{-1} \mathbf{q}^{k}\right)^{T}}{\left(\mathbf{q}^{k}\right)^{T} \mathbf{H}_{k}^{-1} \mathbf{q}^{k}}
$$

where $\mathbf{q}_{k}=\nabla E\left(\mathbf{w}_{k+1}\right)-\nabla E\left(\mathbf{w}_{k}\right)$ is gradient increment and $\mathbf{s}_{k}=\mathbf{w}_{k+1}-\mathbf{w}_{k}$ is variable increment.

\section{Prony Method}

The Prony method is a technique for modelling sampled data as a linear combination of exponential functions [8]. Although it is not a spectral estimation technique, the Prony method has a close relationship to the least squares linear prediction algorithms used for AR and ARMA parameter estimation. Prony method seeks to fit a deterministic exponential model to the data in contrast to AR and ARMA methods that seek to fit a random model to the second-order data statistics.

Assuming $N$ complex data samples the investigated function can be approximated by $p$ exponential functions:

$$
y[n]=\sum_{k=1}^{p} A_{k} e^{\left(\alpha_{k}+j \omega_{k}\right)(n-1) T_{p}+j \psi_{k}}
$$

where

$n=1,2,3, . ., N, T_{p}$ - sampling period, $A_{k}$ - amplitude, $\alpha_{k}$ damping factor, $\omega_{k}$ - angular velocity, $\psi_{k}$ - initial phase.The discrete-time function may be concisely expressed in the form

$$
y[n]=\sum_{k=1}^{p} h_{k} z_{k}^{n-1}
$$

where $h_{k}=A_{k} e^{j \psi_{k}}$ and $z_{k}=e^{\left(\alpha_{k}+j \omega_{k}\right) T_{p}}$.

The estimation problem is based on the minimization of the squared error over the $N$ data values

$$
\delta=\sum_{n=1}^{N}|\varepsilon[n]|^{2}
$$

where

$$
\varepsilon[n]=x[n]-y[n]=x[n]-\sum_{k=1}^{p} h_{k} z_{k}^{n-1}
$$

This turns out to be a difficult nonlinear problem. It can be solved using the Prony method that utilizes linear equation solutions.

If as many data samples are used as there are exponential parameters, then an exact exponential fit to the data can be made.

Consider the $p$-exponent discrete-time function:

$$
x[n]=\sum_{k=1}^{p} h_{k} z_{k}^{n-1}
$$

The $p$ equations of (11) may be expressed in matrix from as

$$
\left[\begin{array}{cccc}
z_{1}^{0} & z_{2}^{0} & \ldots & z_{p}^{0} \\
z_{1}^{1} & z_{2}^{1} & \ldots & z_{p}^{1} \\
\vdots & \vdots & & \vdots \\
z_{1}^{p-1} & z_{2}^{p-1} & \ldots & z_{p}^{p-1}
\end{array}\right] \cdot\left[\begin{array}{c}
h_{1} \\
h_{2} \\
\vdots \\
h_{p}
\end{array}\right]=\left[\begin{array}{c}
x[1] \\
x[2] \\
\vdots \\
x[p]
\end{array}\right]
$$


The matrix equation represents a set of linear equations that can be solved for the unknown vector of amplitudes.

Prony proposed to define the polynomial that has the exponents as its roots:

$$
F(z)=\prod_{k=1}^{p}\left(z-z_{k}\right)=\left(z-z_{1}\right)\left(z-z_{2}\right) \ldots\left(z-z_{p}\right)
$$

The polynomial may be represented as the sum:

$$
\begin{aligned}
& F(z)=\sum_{m=0}^{p} a[m] z^{p-m}= \\
& =a[0] z^{p}+a[1] z^{p-1}+\ldots+a[p-1] z+a[p]
\end{aligned}
$$

Shifting the index on (11) from $n$ to $n-m$ and multiplying by the parameter $a[m]$ yield

$$
a[m] x[n-m]=a[m] \sum_{k=1}^{p} h_{k} z_{k}^{n-m-1}
$$

Equation (14) can be modified into:

$$
\begin{aligned}
& \sum_{m=0}^{p} a[m] x[n-m]= \\
& =\sum_{k=1}^{p} h_{k} z_{k}^{n-p}\left\{\sum_{m=0}^{p} a[m] z_{k}^{p-m-1}\right\}
\end{aligned}
$$

The right-hand summation in (16) may be recognized as a polynomial defined by (14), evaluated at each of its roots yielding the zero result:

$$
\sum_{m=0}^{p} a[m] x[n-m]=0
$$

The equation can be solved for the polynomial coefficients. In the second step the roots of the polynomial defined by (14) can be calculated. The damping factors and sinusoidal frequencies may be determined from the roots $z_{k}$.

For practical situations, the number of data points $N$ usually exceeds the minimum number needed to fit a model of exponentials, i.e. $N>2 p$. In the overdetermined data case, the linear equation (17) should be modified to:

$$
\sum_{m=0}^{p} a[m] x[n-m]=e[n]
$$

The estimation problem is based on the minimization of the total squared error

$$
E=\sum_{n=p+1}^{r}|e[n]|^{2}
$$

\section{Wind generator with capacitors for compensation}

The wind generator with compensating capacitors is shown in Fig.1. The simulation was done in Matlab using the SimPowerSystem Toolbox [9].

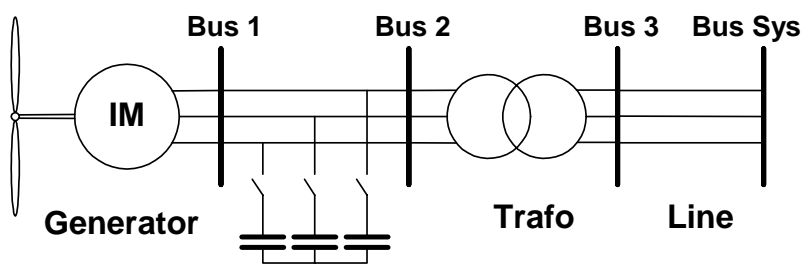

Fig. 1. Induction generator with compensating capacitors

A wind turbine generates power accordingly to mechanical torque on the rotating shaft of the turbine. Produced power depends on rotor speed and pitch angle and is often given in a table form [10].

The pitch control dynamic can be neglected in power system transient analysis [4]. The simulated generator is a $150 \mathrm{~kW}, 400 \mathrm{~V}, 1487 \mathrm{rpm}$, induction machine. It is connected to the grid through a Dyg 25/0.4 $\mathrm{kV}$ distribution transformer which nominal power was varied during the research process and other parameters were set with accordance to [11]. A typical $5 \mathrm{~km}$ overhead line [11] connected the generator to a system. The system was represented by equivalent source with short circuit capacity of $100 \mathrm{MVA}$ and X/R ratio of 7 . The induction generator reactive power demand varies with the produced real power [2]. During the research different compensation levels were simulated. Simulation results correlate with measured values.

\section{Estimation of transients' parameters}

\section{A Analysis of simulated signals}

For variable wind speeds the exact compensation of reactive power using a capacitor bank is difficult [10]. The reactive power variation should be taken into account even if additional capacitors are switched on and off during the operation [7].

Assuming, theoretically, full reactive power compensation at freely chosen wind speed implies capacitance adjustment of the compensating capacitor for every particular case.

Three wind speeds were chosen $-8 \mathrm{~m} / \mathrm{s}, 10 \mathrm{~m} / \mathrm{s}$, $12 \mathrm{~m} / \mathrm{s}$. For these wind speeds the capacitor was adjusted to fully compensate reactive power. Fig. 1 shows the current during the switching and Fig. 3 the FFT of that signal.

Table 1 shows the estimated parameters of signal components of fourth order Prony model (PRO) and obtained using nonlinear regression (NLR). In every case described in this chapter the sampling frequency was $5 \mathrm{kHz}$. Two signal frequencies in the current waveform (Fig. 2) were assumed and estimated. The main $50 \mathrm{~Hz}$ frequency component was named as "No. 2" and an additional capacitor bank switching transient as "No. 1". 
The influence of a capacitor change on transient parameters is clear (Table 1). For higher wind speeds and therefore greater capacitance value, the amplitude of the transients was higher and the transients' frequency lower. Time constant did not change significantly.

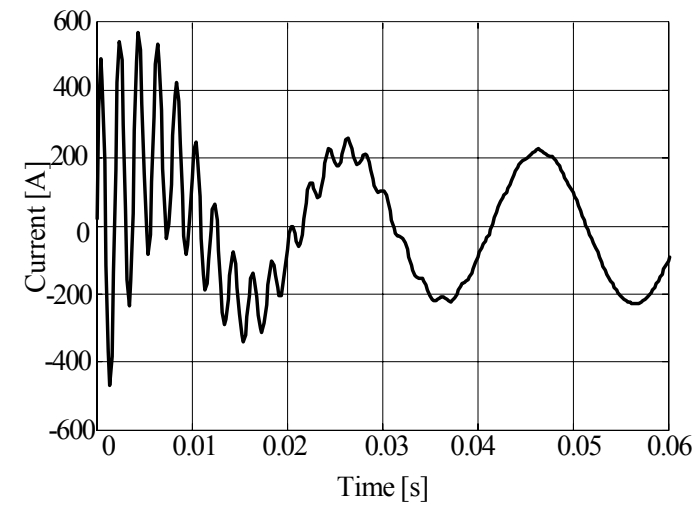

Fig. 2. Current during capacitor bank switching, wind speed $10 \mathrm{~m} / \mathrm{s}$

TABLE I. - Transients' parameters by different wind speed

\begin{tabular}{|c|c|c|c|c|}
\hline $\begin{array}{c}\text { SignalCom./ } \\
\text { Method } \\
\text { [No.-Mth.] }\end{array}$ & $\begin{array}{c}\text { I } \\
\text { [A] }\end{array}$ & $\begin{array}{c}\tau \\
\text { [s] }\end{array}$ & $\begin{array}{c}\text { f } \\
{[\mathbf{H z}]}\end{array}$ & $\begin{array}{c}\boldsymbol{\psi} \\
\text { [rd] }\end{array}$ \\
\hline \multicolumn{5}{|c|}{ wind speed $8 \mathrm{~m} / \mathrm{s}$} \\
\hline 1.-PRO & 579.4 & 0.0095 & 526.6 & -0.15 \\
\hline 1.-NLR & 579.7 & 0.0095 & 526.5 & -0.14 \\
\hline 2.-PRO & 105.7 & 49.66 & -2.37 \\
\hline 2.-NLR & 107.2 & 49.64 & -2.36 \\
\hline \multicolumn{5}{|c|}{ wind speed $10 \mathrm{~m} / \mathrm{s}$} \\
\hline 1.-PRO & 624.8 & 0.0095 & 501.6 & -0.15 \\
\hline 1.-NLR & 625.2 & 0.0095 & 501.6 & -0.15 \\
\hline 2.-PRO & 231.1 & 49.82 & -2.47 \\
\hline 2.-NLR & 233.3 & 49.81 & -2.47 \\
\hline \multicolumn{5}{|c|}{ wind speed $12 \mathrm{~m} / \mathrm{s}$} \\
\hline 1.-PRO & 710.6 & 0.0095 & 456.5 & -0.16 \\
\hline 1.-NLR & 711.1 & 0.0095 & 456.5 & -0.16 \\
\hline 2.-PRO & 394.4 & 49.86 & -2.49 \\
\hline 2.-NLR & 396.7 & 49.86 & -2.48 \\
\hline
\end{tabular}

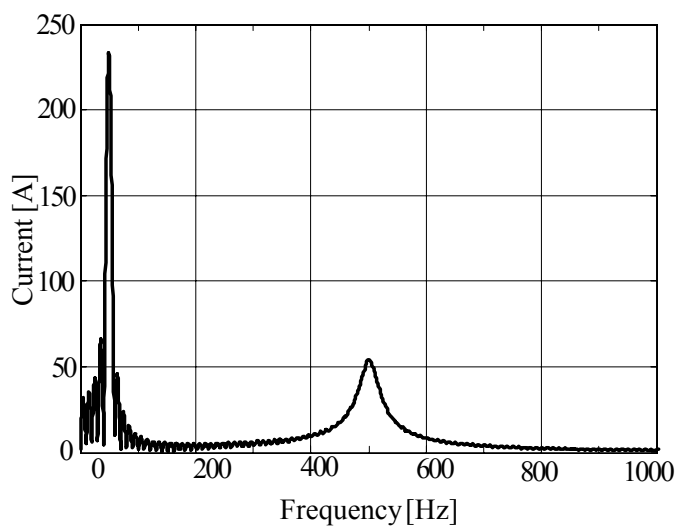

Fig. 3. Fourier transform of the capacitor switching current

\section{B Analysis measured signals}

For variable wind speeds the exact compensation of reactive power using a capacitor bank is difficult [10]. The reactive power variation should be taken into account even if additional capacitors are switched on and off during the operation [7].

Assuming, theoretically, full reactive power compensation at freely chosen wind speed implies capacitance adjustment of the compensating capacitor for every particular case.

Three wind speeds were chosen $-8 \mathrm{~m} / \mathrm{s}, 10 \mathrm{~m} / \mathrm{s}$, $12 \mathrm{~m} / \mathrm{s}$. For these wind speeds the capacitor was adjusted to fully compensate reactive power. Fig. 1 shows the current during the switching and Fig. 3 the FFT of that signal.

Table 1 shows the estimated parameters of signal components of fourth order Prony model (PRO) and obtained using nonlinear regression (NLR). In every case described in this chapter the sampling frequency was $5 \mathrm{kHz}$. Two signal frequencies in the current waveform (Fig. 2) were assumed and estimated. The main $50 \mathrm{~Hz}$ frequency component was named as "No. 2" and an additional capacitor bank switching transient as "No. 1".

The influence of a capacitor change on transient parameters is clear (Table 1). For higher wind speeds and therefore greater capacitance value, the amplitude of the transients was higher and the transients' frequency lower. Time constant did not change significantly.

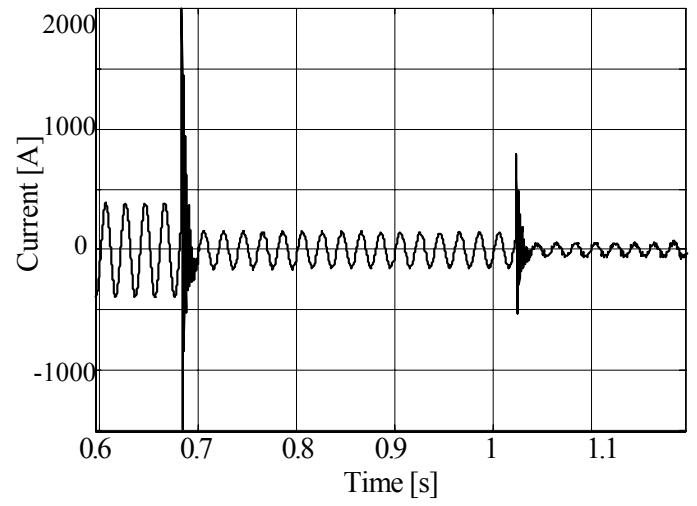

Fig. 4. Current waveform measured during switching of two capacitors

The transients resulting from the both switching operations were analyzed (Table 2 ).

TABLE II. - Transients' parameters computed for switching of two capacitors

\begin{tabular}{|c|c|c|c|c|}
\hline $\begin{array}{c}\text { SignalCom./ } \\
\text { Method } \\
\text { [No.-Mth.] }\end{array}$ & $\begin{array}{c}\mathbf{I} \\
\text { [A] }\end{array}$ & $\begin{array}{c}\tau \\
\text { [s] }\end{array}$ & $\begin{array}{c}\mathbf{f} \\
{[\mathbf{H z}]}\end{array}$ & $\begin{array}{c}\boldsymbol{\Psi} \\
\text { [rd] }\end{array}$ \\
\hline \multicolumn{5}{|c|}{ first capacitor switching } \\
\hline 1.-PRO & 2225.2 & 0.0039 & 592.2 & 0.07 \\
\hline 1.-NLR & 2278.9 & 0.0037 & 592.2 & 0.07 \\
\hline 2.-PRO & 162.9 & & 49.9 & 0.70 \\
\hline 2.-NLR & 155.8 & 49.8 & 0.71 \\
\hline \multicolumn{5}{|c|}{ second capacitor switching } \\
\hline 1.-PRO & 831.0 & 0.0040 & 519.2 & -0.34 \\
\hline 1.-NLR & 849.9 & 0.0039 & 508.4 & -0.22 \\
\hline 2.-PRO & 51.2 & & 50.9 & 0.83 \\
\hline 2.-NLR & 54.2 & & 50.5 & 0.95 \\
\hline
\end{tabular}

Table 2 contains the same symbols and descriptions as Table 1. 


\section{Conclusion}

The research results show, that nonlinear regression and the Prony model are useful for transient estimation in systems with wind generators and compensating capacitors. These methods enabled accurate estimation of amplitude, time constant, phase and frequency of transients' components of simulated and measured signals. The current waveform and its parameters depend on system elements and its operating mode. These dependences could be observed during simulation of various cases.

Application of both Prony model and nonlinear regression required a signal model. Fourier transform, as non parametric method, did not require a signal model or even the number of components, but could not compute signal parameters besides frequency. Using nonlinear regression, the problem of local minima was observed. Unconsidered setting of the initial parameters can lead to local minimum and inadequate results. Additionally, two signal components were predefined, so the rest was considered noise. Application of the Prony method did not show the problem of local minima. The order of the signal model could be easily extended to detect additional components. Both methods delivered similar results, however, application of Prony model seems more suitable for estimation of transients' parameters.

\section{References}

[1] Hammons T.: Status of Integrating renewable electricity production in Europe into the grids. Proceedings of Universities Power Engineering Conference, Cork (Ireland), 2005, p.73

[2] Hanzelka Z., Mroz M., Pawelek R., Pitek K.: Quality Parameters of $15 \mathrm{kV}$ supply voltage after connection of wind farms-case study. Proceedings of Conference on Harmonics and Quality of Power, Cascais (Portugal), September 2006, on CD, paper ID 124

[3] Quinonez-Varela G., Cruden A., Grant A. D., Castaneda A.: Electrical Integration Assessment of Wind Turbines into Industrial Power System: The Case of a Mining Unit. Proceedings IEEE Power Tech Conference, Porto (Portugal), September 2001, paper ID DRS3-292

[4] Chompoo-inwai Ch., Wei-Jen L., Fuangfoo P., Williams M., Liao J. R.: System Impact Study for the Interconection of Wind Generation and Utility System. IEEE Trnas. on Industry Applications, vol. 41, January 2005, pp 163-168

[5] Shulz, D., Moutawakkil, K., Hanitsh, R., Saniter, C: Power Quality Behaviour of Large Wind Parkswith variable speed wind energy converters. CIRED, $17^{\text {th }}$ International Conference on Electricity Distribution, Barcelona, Spain, 12-15 Mai 2003, CD

[6] Tabesh A., Iravani R.: Transient Behavior of FixedSpeed Grid-Connected Wind Farm. Transactions of International Conference on Power Systems Transients, Montreal (Canada), June 2005, Paper No. IPST05-068

[7] Thiringer T.: Power Quality Measurments Performed on a Low-Voltage Grid Equipped with Two Wind
Turbines. IEEE Trans. on Energy Conversion, vol. 11, September 1996, pp. 601-606

[8] Lobos T., Rezmer J., Kolin H.-J.: Analysis of Power System Transients using Wavelets and Prony Method. Proceedings IEEE Power Tech Conference, Porto (Portugal), September 2001, paper ID EMT-103

[9] The Matworks: SimPowerSystems User's Guide. The Math Works Inc., 2006

[10] Lubosny Z.: Wind Turbine Operation in Electric Power Systems. Springer Verlag, Berlin Heidelberg (Germany), 2003

[11] SIEMENS: Electrical Engineering Handbook, 1987

[12] Bhatti A. M.: Practical OptimizationMethods. Springer Verlag, New York (USA), 2000, pp. 288302

[13] Larsson A. Thiringer T.: Measurment on and Modelling of Capacitor-Connecting Transients on a Low-voltage Grid Equipped with Two Wind Turbines. Proceedings IPST Conference, Lisbon (Portugal), 1995 